\title{
BMJ Open Which biopsychosocial factors are associated with the development of chronic musculoskeletal pain? Protocol for an umbrella review of systematic reviews
}

\author{
Michael Dunn (D) , ${ }^{1,2}$ Alison B Rushton (D) ," Jai Mistry (D) ,,3 Andrew Soundy (D) ,' \\ Nicola R Heneghan (D) ${ }^{1}$
}

To cite: Dunn M, Rushton AB, Mistry J, et al. Which biopsychosocial factors are associated with the development of chronic musculoskeletal pain? Protocol for an umbrella review of systematic reviews. BMJ Open 2021;11:e053941. doi:10.1136/ bmjopen-2021-053941

- Prepublication history and additional supplemental material for this paper are available online. To view these files, please visit the journal online (http://dx.doi.org/10.1136/ bmjopen-2021-053941).

Received 03 August 2021 Accepted 28 September 2021

\section{Check for updates}

(C) Author(s) (or their employer(s)) 2021. Re-use permitted under CC BY-NC. No commercial re-use. See rights and permissions. Published by BMJ.

${ }^{1}$ School of Sport, Exercise and Rehabilitation Sciences, University of Birmingham, Birmingham, UK

${ }^{2}$ Musculoskeletal Physiotherapy, St George's University Hospitals NHS Foundation Trust, London, UK

${ }^{3}$ School of Physical Therapy, Western University Faculty of Health Sciences, London, Ontario, Canada

Correspondence to Michael Dunn;

Michael.Dunn@stgeorges. nhs.uk

\section{ABSTRACT}

Introduction Recent systematic reviews have identified many biopsychosocial factors associated with the development of chronic musculoskeletal pain (CMP). Despite often being specific to a particular musculoskeletal condition, findings are similar across systematic reviews. Research is needed to aggregate these findings to identify consistent factors across musculoskeletal disorders that are associated with the development of CMP. The objective of this study is to provide a meta-level synthesis of all biopsychosocial factors associated with the development of CMP.

Methods and analysis An umbrella review and metalevel narrative synthesis \pm meta-analysis has been designed informed by Joanna Briggs Institute and Cochrane guidance. This protocol is reported in line with the Preferred Reporting Items for Systematic Reviews and Meta-Analysis-P. Sources will include Ovid Medline, Embase, Web of Science Core Collection, Cochrane Database of Systematic Reviews, Database of Abstracts of Reviews of Effects, PsycINFO, CINAHL, PEDro, PROSPERO, Google Scholar and grey literature. Inclusion criteria: any systematic review which investigates biopsychosocial factors which may be associated with the development of CMP through prospective longitudinal methods. The outcome is musculoskeletal pain lasting beyond 3 months. Two independent reviewers will be involved in all stages; screening, selection, data extraction and risk of bias evaluation using the Assessing the Methodological Quality of Systematic Reviews-2 guidelines. A metalevel narrative synthesis will be conducted based on (a) factors associated with development of CMP, (b) the range of musculoskeletal disorders for which the same/similar findings have been established and (c) the quality of studies informing these findings. Where possible, meta-analysis will be performed. The Grading of Recommendations, Assessment, Development and Evaluation guidelines will be followed to determine the level of evidence for each biopsychosocial factor.

Ethics and dissemination This umbrella review does not require ethical approval. Findings will be presented at conferences and published in a peer reviewed journal. PROSPERO registration number CRD42020193081.

\section{Strength and limitations on this study}

- Inclusion of a substantial number of original research papers and participants.

- The inclusion of all musculoskeletal conditions will establish findings pertinent to a great number of stakeholders and clinical decision-makers.

- The methods employed for this review are rigorous and in line with the most relevant and up to date Cochrane and Joanna Briggs Institute Guidance.

- Quality of life and disability are common outcomes for systematic reviews of chronic musculoskeletal pain but are not included in this review.

- This umbrella review will be limited to studies whereby the full text is available in the English language.

\section{INTRODUCTION}

The International Classification of Diseases (ICD) describes chronic musculoskeletal pain (CMP) as pain that arises as part of a disease process directly affecting bones, joints, muscle or related soft tissue lasting longer than 3 months. ${ }^{1}$ CMP arises secondary to a complex interaction between the systems of the body including the nervous, endocrine, immunological, autonomic, psychological and musculoskeletal (MSK) systems. This interaction creates a maintained elevated perception of threat within the nociceptive system; thus facilitating a prolonged or repeated experience of pain despite limited evidence of potential or actual tissue damage. ${ }^{2-5}$ This transition to a sensitised nociceptive system, termed 'nociplastic pain' by the International Association for the Study of Pain, is thought to explain the mechanisms of CMP. ${ }^{6}$

In the UK, the National Health Service spent $£ 4.7$ billion pounds on MSK conditions in $2015^{7}$-an annual figure which is likely 
higher now—causing a loss over 30 million working days each year due to MSK conditions, ${ }^{8}$ creating a substantial financial and economic burden. MSK complaints also account for up to $30 \%$ of general practitioner consultations. ${ }^{9}$ A significant proportion of these likely being for the management of CMP with its estimated prevalence in the UK at $43 \%{ }^{10} \mathrm{CMP}$ is also a huge burden internationally with low back pain identified as the single greatest cause of years lived with a disability; ${ }^{11}$ thus prompting the ICD to recently recognise CMP as a disease in its own right. ${ }^{1}$ Given the apparent difficulty in effectively managing CMP, and its increasing prevalence, ${ }^{12}$ a better understanding of CMP is needed.

To better understand CMP, there is a need to understand the factors which influence its development. The experience of MSK pain is biopsychosocial and therefore biological factors, such as the type and severity of MSK condition, do not equate to consistent experiences of pain among individuals. ${ }^{13-15}$ Rather, many individuals go on to develop CMP where others do not. This discrepancy has frequently been associated with the presence of many biopsychosocial factors such as catastrophised beliefs, lower socioeconomic status and fear of movement. ${ }^{16-18}$ However, existing systematic reviews often focus on biological, psychological or social factors individually or in relation to only one form or CMP, for example, low back pain. Given that nociplastic mechanisms, and many biopsychosocial factors, are not specific to a particular MSK condition, synthesis of this evidence base may inform future research and management of CMP conditions as a whole.

Biomedical healthcare approaches tend to dominate the management of MSK conditions with biopsychosocial approaches often only considered once medical management has failed, as demonstrated by National Institute for Health and Care Excellence guidance for low back pain. ${ }^{19}$ This equates to a reactionary approach to CMP whereby biopsychosocial care is usually only implemented once CMP is well established. By this point, CMP is very difficult to effectively treat with $79 \%-92 \%$ of people still experiencing pain when followed-up up to 12 years later. ${ }^{20-22}$ A synthesis of the biopsychosocial factors associated with the development of CMP may help identify those at risk of CMP before it has been established. This provides a window of opportunity for healthcare services to deliver proactive biopsychosocial treatments with an aim of preventing development of CMP. This strategy works well for other chronic diseases such as heart disease ${ }^{23}$ and diabetes. ${ }^{24}$

To address the growing prevalence and burden of CMP, it is timely to synthesise the existing literature to identify which biopsychosocial factors are associated with its development. Given the abundance of systematic reviews, an umbrella review will be most appropriate in achieving this.

\section{AIM}

To establish which biopsychosocial factors are associated with the development of CMP.

\section{METHODS}

\section{Design and registration}

This protocol was developed in accordance with the Preferred Reporting Items for Systematic Reviews and Meta-Analyses Protocol (PRISMA-P) ${ }^{25}$ (see online supplemental file A for completed PRISMA-P checklist). The methodology has been guided by the Joanna Briggs Institute Manual for Evidence Synthesis of Umbrella Reviews ${ }^{26}{ }^{27}$ and the Cochrane handbook for the conduct of systematic reviews. ${ }^{28}$

\section{Patient and public involvement}

The basis for the need and relevance of this umbrella review is based on the clinical interactions of the authors with previous and current patients with CMP. This includes members of the patient and public involvement and engagement group within the Centre of Precision Rehabilitation for Spinal Pain at the University of Birmingham. The group includes individuals with chronic spinal pain.

\section{Eligibility criteria}

The eligibility criteria were established using a modified PICOS statement (see table 1). 'I' was replaced with 'E'

\begin{tabular}{ll}
\hline Table 1 PICOS statement \\
\hline $\begin{array}{l}\text { Population } \\
\text { Exposure }\end{array}$ & $\begin{array}{l}\text { Any biopsychosocial factors which may have influenced outcome, for example, patient reported higher } \\
\text { levels of pain at onset. }\end{array}$ \\
Comparator & $\begin{array}{l}\text { Those who have not been exposed to the biopsychosocial factor under investigation, for example, the } \\
\text { comparator to those who report higher levels of pain at onset would be those who report lower levels of } \\
\text { pain at onset. }\end{array}$ \\
Outcome & $\begin{array}{l}\text { Persistence in MSK pain beyond } 3 \text { months. This may be identified through any MSK pain-related outcome } \\
\text { measure or through clinician diagnosis. }\end{array}$ \\
Study designs & Systematic reviews of prospective longitudinal design studies.
\end{tabular}

MSK, musculoskeletal. 
for exposure due to the epidemiological nature of the review. $^{29}$

\section{Inclusion criteria}

Systematic reviews of observational studies with/without meta-analysis investigating factors associated with MSK pain lasting longer than 3 months.

\section{Exclusion criteria}

Systematic reviews which include interventional studies, for example, factors associated with successful surgery, populations with a high risk or evidence of poor tissue healing, for example, autoimmune disorders, injuries where tissue healing may be incomplete at 3 months, for example, fractures, draw body region-specific conclusions which are not generalisable to the wider CMP population, for example, a bony heel spur associated with chronic heel pain, pool data with non-MSK chronic pain populations, for example, cancer-related pain, potential systemic drivers of CMP, for example, spondyloarthropathy, do not include an appropriate comparator and systematic reviews where the full text is not available in the English language.

\section{Information sources}

A systematic search of electronic databases and grey literature will be conducted. Ovid MEDLINE, Ovid EMBASE, Web of Science Core Collection and Google Scholar will be searched as suggested by Bramer et al, ${ }^{30}$ who found this combination to be most effective for the retrieval of systematic reviews. In addition, the PEDro, CINAHL and Ovid PsycINFO databases will be searched as well as the Cochrane database for systematic reviews, the Database of Abstracts of Reviews of Effects and the PROSPERO register as all are particularly relevant for the subject of MSK pain and/or systematic reviews. There will be no limitation on search dates. Grey literature will be searched using the Canadian Agency for Drugs and Technologies in Health grey literature searching tool. To ensure literature saturation, we will also scan the reference lists of included studies or relevant reviews identified through the search.

\section{Search strategy}

The search strategy has been designed by the lead author (MD) with the assistance of coauthors (NRH, ABR and AS) and an experienced health sciences librarian with expertise of systematic review searching. The search strategy includes a validated search strategy for prognostic studies recommended by the Cochrane Prognosis Research Group. ${ }^{31}$ The initial search strategy was developed with Ovid MEDLINE using medical subject headings and text words and subsequently adapted to the syntax and subject headings of the other databases to be searched for this review. The search strategy has been peer reviewed by other members of the review team (NRH, $\mathrm{ABR}, \mathrm{AS}$ and $\mathrm{JM}$ ) to reduce the risk of search errors and ensure the highest retrieval of eligible studies. ${ }^{32}$ Please see online supplemental file B for the Ovid MEDLINE search strategy.

\section{Screening and selection}

A systematic search of databases using the search strategies will be conducted by two reviewers independently of each other (MD and JM). Titles and abstracts will be retrieved and stored using Microsoft EndNote X9.3.3, will be assessed based on the inclusion/exclusion criteria and allocated into one of two groups: potentially eligible or not eligible. Full-text sources for potentially eligible studies will then be sourced and discussed between both reviewers for confirmation of eligibility. Additional information to determine eligibility will be sought from study authors via email where necessary. Any disagreement of eligibility at this stage will be referred to a third reviewer (NRH). All reasons for excluding reviews will be recorded using a PRISMA flow chart. None of the reviewers will be blinded to the journal titles, study authors or institutions.

\section{Data extraction}

Two reviewers (MD and JM) will independently extract data. All data excluded will be agreed by both reviewers in discussion and any disagreement will be referred to a third reviewer (NRH). Where study data are unclear or missing, the corresponding author will be contacted via email for clarification or further information. A second and final email will be sent if no response has been received after 2 weeks. In the event of a non-response after a further 2 weeks, includable data will be extracted based on agreement between the two reviewers with any disagreement referred to a third reviewer. Data will be extracted using a bespoke and standardised proforma which has been piloted a priori (see online supplemental file C). Data extraction will, where possible, include quantitative data such as demographic information, ORs, relative risk ratios, size of effects and Pearsons $\chi^{2}$ scores which will be stored on Microsoft Excel.

\section{Data items}

The data to be extracted are summarised in table 2. As per Joanna Briggs Umbrella Review guidance, data will not be collected from the original research studies but extracted from the included systematic reviews only.

\section{Risk of bias assessment}

Two reviewers (MD and JM) will independently perform risk of bias assessment of included systematic reviews using the Assessing the Methodological Quality of Systematic Reviews-2 (AMSTAR-2) guidelines and checklist. ${ }^{33}$ AMSTAR-2 has been shown to be valid and reliable for the appraisal of systematic reviews ${ }^{34} 35$ and has been used in many high-quality healthcare Umbrella Reviews. ${ }^{36-38}$ AMSTAR-2 includes assessment of study eligibility criteria, identification and selection of studies, data collection methods, study appraisal methods and findings and synthesis methods. Overall confidence in the results of each systematic review will be rated as high, moderate, low or critically low. ${ }^{33}$ Any disagreements will be discussed 
Table 2 Summary of items to be extracted from included studies

Study and population

characteristics

Outcome measures

Methods and findings
Reference, type of population (eg, idiopathic, traumatic, mixed), number of studies included, number of participants and demographic information. Name of outcome measures or methods used to determine CMP.

Method of data synthesis, biopsychosocial factors associated with development of CMP, methods of data collection for biopsychosocial factors, musculoskeletal conditions for which findings have been established, quality of studies informing findings, appraisal tools used and quantitative results including but not limited to ORs and relative risk ratios.

CMP, chronic musculoskeletal pain.

and referred to a third reviewer (NRH) for a conclusive decision. Reporting bias will be determined based on whether a protocol exists as a peer review publication or through registration with PROSPERO.

\section{Data synthesis}

Meta-analysis will be performed where two or more included reviews have reported on the same biopsychosocial factor and the data can be suitably pooled for statistical analysis. Statistical analysis will then be performed by computing OR using a random-effects model with a $95 \%$ CI. Pearson's $\chi^{2}$ test will be used to assess statistical significance of heterogeneity between reviews. Publication bias will be assessed through the use of an inverted funnel plot. All statistical analysis will be conducted using IBM SPSS Statistics V.26 and Microsoft Excel.

Where meta-analysis is not possible, meta-level narrative synthesis will be performed in accordance with guidance by Popay et al (Guidance on the Conduct of Narrative Synthesis in Systematic Reviews. Results of an ESRC funded research project (unpublished report)) and The Cochrane Consumers and Communication Review Group. ${ }^{39}$ However, the first step of 'developing a theory of how the intervention works' will be removed due to the non-interventional nature of this umbrella review. Narrative synthesis will be structured around three domains: (\#a) the biological, psychological or social factors associated with the development of CMP, (b) the range of MSK disorders for which the same/similar findings have been established and (c) the risk of bias of the studies informing these findings. Using standardised templates, data will be extracted and presented in tabular format. Discussion will be orientated around similarities and differences between the findings of included studies, in line with our three domains. A detailed outline of methodological problems or biases will also be included, alongside an assessment of completeness and applicability. ${ }^{39}$

\section{Confidence in cumulative evidence}

Umbrella reviews have been criticised for lack of consistent or appropriate methods in ascertaining the level of certainty of findings. ${ }^{40}$ The Grading of Recommendations Assessment, Development and Evaluation (GRADE) approach is a well-established tool which is commonly used for this purpose with systematic reviews and has been recommended for use with umbrella reviews. ${ }^{40}$ The
GRADE approach has been adapted for the assessment of evidence of prognostic factors. ${ }^{41}$ GRADE enables evaluation of criteria rating down factors associated with the development of CMP (risk of bias (informed by AMSTAR2), inconsistency, indirectness, imprecision and publication bias) and criteria of rating up factors associated with the development of CMP (dose-response, large effect or for the nature of plausible biases) to provide a statement of the overall level of evidence as high, moderate, low or very low for each biopsychosocial factor.

\section{DISCUSSION}

Traditionally, research of CMP has been orientated towards a particular chronic MSK condition rather than considering CMP as an overarching condition in itself. Rather, this umbrella review will consider CMP as a disease in its own right in line with the $\mathrm{ICD}^{1}$ and seeks to contribute to the body of evidence supporting a shift away from the over-medicalisation of CMP. ${ }^{42}$

Physical assessment and imaging are typically utilised to determine physical 'abnormalities' with these 'abnormalities' then serving as the rationale for the diagnosis and treatment of a specific MSK condition. ${ }^{43-45}$ Treatments may include surgery, acupuncture, manual therapy, injections, pharmacology or surgery ${ }^{19}$ among others, with the aim of 'fixing' the 'abnormality' and improving the MSK condition. However, research demonstrates that these 'abnormalities' are heavily prevalent in healthy populations with no history of MSK conditions. ${ }^{43} 46$ This raises concern for the validity of this form of diagnosis. In addition, a large multicentre randomised control trial also demonstrates that sham surgery is equally as effective as surgical intervention for shoulder pain, ${ }^{48}$ raising questions for the mechanisms of how effective interventions for MSK pain work. This same finding has also been found with sham surgery for knee, back and elbow pain as evidenced by a high-quality systematic review ${ }^{49}$ suggesting that the mechanisms of pain and successful intervention are similar, regardless of the type of MSK condition. Notably, the participants in these studies typically have had symptoms for longer than 3 months and therefore these findings are relevant to the CMP population.

The nociplastic mechanisms by which individuals experience CMP are thought to be contributed to by functional 
and anatomical changes in the central nervous system. ${ }^{50-52}$ Reorganisation of brain activity takes place with reduced activation of the somatosensory cortex, which is usually highly active during nociception, and an increase in activity of the corticolimbic system, thus creating a shift from nociceptive to emotional circuits. ${ }^{50}$ These changes have been shown to be consistent across a range of MSK conditions. ${ }^{53-56}$ Furthermore, this persistent activity of the corticolimbic system is responsible for the development of many behavioural and psychological presentations,${ }^{57}$ many of which have separately been identified as risk factors for the development of CMP, such as fear. ${ }^{16} 18$ This is further evidenced by the consistent biopsychosocial factors shown to be associated with development of CMP regardless of the type of MSK condition. ${ }^{16-18}$

For these reasons, and that CMP is considered a disease in its own right, this umbrella review recognises the need to aggregate the findings of systemic reviews on the biopsychosocial factors associated with the development of CMP, for all MSK conditions.

\section{AMENDMENTS}

In the event of protocol amendments, the date of each amendment will be accompanied by a description of the change and the rationale for the change.

Twitter Michael Dunn @MikeDunnPhysio, Alison B Rushton @abrushton, Jai Mistry @JaiMistry9, Andrew Soundy @Andy_Soundy and Nicola R Heneghan @ HeneghanNicola

Contributors MD conceived, designed and will coordinate the review; will collect, manage, analyse and interpret data; and write the review. JM will collect, manage analyse and interpret data. NRH, ABR and AS contributed to conception and design of the review and will provide general advice on the coordination of the review as well as collection, management, analysis and synthesis of data.

Funding The authors have not declared a specific grant for this research from any funding agency in the public, commercial or not-for-profit sectors.

Competing interests None declared.

Patient consent for publication Not required.

Provenance and peer review Not commissioned; externally peer reviewed.

Supplemental material This content has been supplied by the author(s). It has not been vetted by BMJ Publishing Group Limited (BMJ) and may not have been peer-reviewed. Any opinions or recommendations discussed are solely those of the author(s) and are not endorsed by BMJ. BMJ disclaims all liability and responsibility arising from any reliance placed on the content. Where the content includes any translated material, BMJ does not warrant the accuracy and reliability of the translations (including but not limited to local regulations, clinical guidelines, terminology, drug names and drug dosages), and is not responsible for any error and/or omissions arising from translation and adaptation or otherwise.

Open access This is an open access article distributed in accordance with the Creative Commons Attribution Non Commercial (CC BY-NC 4.0) license, which permits others to distribute, remix, adapt, build upon this work non-commercially, and license their derivative works on different terms, provided the original work is properly cited, appropriate credit is given, any changes made indicated, and the use is non-commercial. See: http://creativecommons.org/licenses/by-nc/4.0/.

\section{ORCID iDs}

Michael Dunn http://orcid.org/0000-0002-6559-663X

Alison B Rushton http://orcid.org/0000-0001-8114-7669

Jai Mistry http://orcid.org/0000-0002-2057-0380

Andrew Soundy http://orcid.org/0000-0002-5118-5872

Nicola R Heneghan http://orcid.org/0000-0001-7599-3674
REFERENCES

1 Treede R-D, Rief W, Barke A, et al. A classification of chronic pain for ICD-11. Pain 2015;156:1003-7.

2 Gifford L. Pain, the tissues and the nervous system: a conceptual model. Physiotherapy 1998;84:27-36.

3 Melzack R. Evolution of the neuromatrix theory of pain. The Prithvi Raj Lecture: presented at the third World Congress of World Institute of pain, Barcelona 2004. Pain Pract 2005;5:85-94.

4 Moseley GL, Gallace A, Spence C. Bodily illusions in health and disease: physiological and clinical perspectives and the concept of a cortical 'body matrix'. Neurosci Biobehav Rev 2012;36:34-46.

5 Melzack R, Katz J. Pain. Wiley Interdiscip Rev Cogn Sci 2013;4:1-15.

6 Kosek E, Cohen M, Baron R, et al. Do we need a third mechanistic descriptor for chronic pain states? Pain 2016;157:1382-6.

7 NHS England. CCG programme budgeting benchmarking tool 2013/14. Available: https://www.england.nhs.uk/wp-content/ uploads/2015/06/13-14-ccg-prog-bug-benchmarking-tool.xlsm [Accessed 02 Jun 2020].

8 Office for National Statistics. Sickness absence in the UK labour market, 2016. Available: https://www.ons.gov.uk/employmentandlab ourmarket/peopleinwork/labourproductivity/articles/sicknessabse nceinthelabourmarket/2016 [Accessed 16 Jul 2020].

9 Department of Health. The musculoskeletal services framework - a joint responsibility: doing it differently. The musculoskeletal services framework, 2006. Available: https://webarchive.nationalarchives.gov. uk/20130107105354/http:/www.dh.gov.uk/prod_consum_dh/groups/ dh_digitalassets/@dh/@en/documents/digitalasset/dh_4138412.pdf [Accessed 02 June 2020].

10 Fayaz A, Croft P, Langford RM, et al. Prevalence of chronic pain in the UK: a systematic review and meta-analysis of population studies. BMJ Open 2016;6:e010364.

11 Rice ASC, Smith BH, Blyth FM. Pain and the global burden of disease. Pain 2016;157:791-6.

12 Vos T, Flaxman AD, Naghavi M, et al. Years lived with disability (YLDs) for 1160 sequelae of 289 diseases and injuries 1990-2010: a systematic analysis for the global burden of disease study 2010 . Lancet 2012;380:2163-96.

13 Chapman WP, Jones CM. Variations in cutaneous and visceral pain sensitivity in normal subjects. J Clin Invest 1944;23:81-91.

14 Nielsen CS, Staud R, Price DD. Individual differences in pain sensitivity: measurement, causation, and consequences. J Pain 2009;10:231-7.

15 Yarnitsky D, Granot M, Granovsky Y. Pain modulation profile and pain therapy: between pro- and antinociception. Pain 2014;155:663-5.

16 Pincus T, Burton AK, Vogel S, et al. A systematic review of psychological factors as predictors of chronicity/disability in prospective cohorts of low back pain. Spine 2002;27:E109-20.

17 Hagen K, Zwart J-A, Svebak S, et al. Low socioeconomic status is associated with chronic musculoskeletal complaints among 46,901 adults in Norway. Scand J Public Health 2005;33:268-75.

18 Martinez-Calderon J, Meeus M, Struyf F, et al. The role of psychological factors in the perpetuation of pain intensity and disability in people with chronic shoulder pain: a systematic review. BMJ Open 2018;8:e020703.

19 National Institute for Health and Care Excellence. Low back pain and sciatica in over 16S: assessment and management. Available: www. nice.org.uk/guidance/ng59 [Accessed 02 June 2020].

20 Elliott AM, Smith BH, Hannaford PC, et al. The course of chronic pain in the community: results of a 4 -year follow-up study. Pain 2002;99:299-307.

21 Andersson HI. The course of non-malignant chronic pain: a 12year follow-up of a cohort from the general population. Eur J Pain 2004;8:47-53.

22 Landmark T, Dale O, Romundstad P, et al. Development and course of chronic pain over 4 years in the general population: The HUNT pain study. Eur J Pain 2018;22:1606-16.

23 Puska P, Ståhl T. Health in all policies-the Finnish initiative: background, principles, and current issues. Annu Rev Public Health 2010;31:315-28.

24 Diabetes Prevention Program Research Group. 10 year follow up of diabetes incidence and weight loss in the diabetes prevention program outcomes study. Lancet 2010;374:1677-86.

25 Shamseer L, Moher D, Clarke M, et al. Preferred reporting items for systematic review and meta-analysis protocols (PRISMA-P) 2015: elaboration and explanation. BMJ 2015;349:g7647.

26 Aromataris E, Fernandez R, Godfrey CM, et al. Summarizing systematic reviews: methodological development, conduct and reporting of an umbrella review approach. Int J Evid Based Healthc 2015;13:132-40. 
27 Aromataris E, Fernandez R, Godfrey CM. Chapter 10: Umbrella Reviews. In: Aromataris E, Munn Z, eds. JBI manual for evidence synthesis, 2020.

28 Higgins J, Green S. Cochrane Handbook for systematic reviews of interventions. Available: https://handbook-5-1.cochrane.org/chapter 7/7_2_4_implementation_of_the_selection_process.htm [Accessed 02 Jun 2020]

29 McKenzie JE, Brennan SE, Ryan RE. Chapter 3: Defining the criteria for including studies and how they will be grouped for synthesis. In: Cochrane Handbook for systematic reviews of interventions version 6.1, 2020. www.training.cochrane.org/handbook

30 Bramer WM, Rethlefsen ML, Kleijnen J, et al. Optimal database combinations for literature searches in systematic reviews: a prospective exploratory study. Syst Rev 2017;6:245.

31 Geersing G-J, Bouwmeester W, Zuithoff P, et al. Search filters for finding prognostic and diagnostic prediction studies in MEDLINE to enhance systematic reviews. PLoS One 2012;7:e32844.

32 McGowan J, Sampson M, Salzwedel DM, et al. PRESS Peer Review of Electronic Search Strategies: 2015 Guideline Statement. J Clin Epidemiol 2016;75:40-6.

33 Shea BJ, Reeves BC, Wells G, et al. AMSTAR 2: a critical appraisal tool for systematic reviews that include randomised or nonrandomised studies of healthcare interventions, or both. $B M J$ 2017;358:j4008.

34 Lorenz RC, Matthias K, Pieper D, et al. A psychometric study found AMSTAR 2 to be a valid and moderately reliable appraisal tool. $J$ Clin Epidemiol 2019;114:133-40.

35 Pieper D, Puljak L, González-Lorenzo M, et al. Minor differences were found between AMSTAR 2 and ROBIS in the assessment of systematic reviews including both randomized and nonrandomized studies. J Clin Epidemiol 2019;108:26-33.

36 Miller P, Soundy A. The pharmacological and non-pharmacological interventions for the management of fatigue related multiple sclerosis. J Neurol Sci 2017:381:41-54.

37 Thomson K, Hillier-Brown F, Todd A, et al. The effects of public health policies on health inequalities in high-income countries: an umbrella review. BMC Public Health 2018;18:869.

38 Martinez-Calderon J, Flores-Cortes M, Morales-Asencio JM, et al. Which psychological factors are involved in the onset and/ or persistence of musculoskeletal pain? an umbrella review of systematic reviews and meta-analyses of prospective cohort studies. Clin J Pain 2020;36:626-37.

39 Ryan R. Cochrane Consumers and Communication Review Group. 'Cochrane Consumers and Communication Review Group: data synthesis and analysis'. Available: http://cccrg.cochrane.org [Accessed 02 June 2020].

40 Schlesinger S, Schwingshackl L, Neuenschwander M, et al. A critical reflection on the grading of the certainty of evidence in umbrella reviews. Eur J Epidemiol 2019;34:889-90.
41 Foroutan F, Guyatt G, Zuk V, et al. Grade guidelines 28: use of grade for the assessment of evidence about prognostic factors: rating certainty in identification of groups of patients with different absolute risks. J Clin Epidemiol 2020;121:62-70.

42 Lewis JS, Cook CE, Hoffmann TC, et al. The elephant in the room: too much medicine in musculoskeletal practice. J Orthop Sports Phys Ther 2020;50:1-4.

43 Lewis JS, Green A, Wright C. Subacromial impingement syndrome: the role of posture and muscle imbalance. J Shoulder Elbow Surg 2005:14:385-92.

44 Li ALK, Yen D. Effect of increased MRI and CT scan utilization on clinical decision-making in patients referred to a surgical clinic for back pain. Can J Surg 2011;54:128-32.

45 Braman JP, Zhao KD, Lawrence RL, et al. Shoulder impingement revisited: evolution of diagnostic understanding in orthopedic surgery and physical therapy. Med Biol Eng Comput 2014;52:211-9.

46 Brinjikji W, Luetmer PH, Comstock B, et al. Systematic literature review of imaging features of spinal degeneration in asymptomatic populations. AJNR Am J Neuroradiol 2015;36:811-6.

47 Barreto RPG, Braman JP, Ludewig PM, et al. Bilateral magnetic resonance imaging findings in individuals with unilateral shoulder pain. J Shoulder Elbow Surg 2019;28:1699-706.

48 Beard DJ, Rees JL, Cook JA, et al. Arthroscopic subacromial decompression for subacromial shoulder pain (CSAW): a multicentre, pragmatic, parallel group, placebo-controlled, three-group, randomised surgical trial. Lancet 2018;391:329-38.

49 Louw A, Diener I, Fernández-de-Las-Peñas C, et al. Sham surgery in Orthopedics: a systematic review of the literature. Pain Med 2017:18:736-50.

50 Hashmi JA, Baliki MN, Huang L, et al. Shape shifting pain: chronification of back pain shifts brain representation from nociceptive to emotional circuits. Brain 2013;136:2751-68.

51 Yu R, Gollub RL, Spaeth R, et al. Disrupted functional connectivity of the periaqueductal gray in chronic low back pain. Neuroimage Clin 2014;6:100-8.

52 McCarberg B, Peppin J. Pain pathways and nervous system plasticity: learning and memory in pain. Pain Med 2019;20:2421-37.

53 Mutso AA, Radzicki D, Baliki MN, et al. Abnormalities in hippocampal functioning with persistent pain. J Neurosci 2012;32:5747-56.

54 Kyle BN, McNeil DW. Autonomic arousal and experimentally induced pain: a critical review of the literature. Pain Res Manag 2014:19:159-67.

55 Apkarian AV, Mutso AA, Centeno MV, et al. Role of adult hippocampal neurogenesis in persistent pain. Pain 2016;157:418-28.

56 Yuan C, Shi H, Pan P, et al. Gray matter abnormalities associated with chronic back pain. Clin J Pain 2017;33:983-90.

57 Yang S, Chang MC. Chronic pain: structural and functional changes in brain structures and associated negative affective states. Int $\mathrm{J} \mathrm{Mol}$ Sci 2019;20:3130. 